\title{
Liquor Habit Transmission Model
}

\author{
Nita H. Shah', Bijal M. Yeolekar'1, Nehal J. Shukla ${ }^{2}$ \\ ${ }^{1}$ Department of Mathematics, Gujarat University, Ahmedabad, India \\ ${ }^{2}$ Department of Mathematics, Columbus State University, Columbus, USA \\ Email: nitahshah@gmail.com, bijalyeolekar28@gmail.com, shukla nehal@columbusstate.edu
}

Received 28 May 2015; accepted 7 July 2015; published 10 July 2015

Copyright (C) 2015 by authors and Scientific Research Publishing Inc.

This work is licensed under the Creative Commons Attribution International License (CC BY). http://creativecommons.org/licenses/by/4.0/

(c) (i) Open Access

\begin{abstract}
In this paper, we analyse population dynamics of liquor habit. Liquor free and Liquor endemic equilibrium are computed. The local and global stabilities of the proposed problem are established. The numerical simulation is given to validate the transmission of population indifferent compartment using state-space model.
\end{abstract}

\section{Keywords}

Liquor Habit, Basic Reproduction Number, Local Stability, Global Stability, State-Space Simulation

\section{Introduction}

The Ayurvedas depicted that the alcohol behave as a medicine if it is taken for the purpose of meditation otherwise it behave as a poison if it is taken in addiction manner for the humans. The liquor habits reflect person's social status and cultural prestige. It is observed that transmission of liquoring spreads frequently these days due to availability of the liquor in the market. There is a similarity between spread of infectious disease and liquor habits. In other words, liquor habit can be treated as a virus which transmits among the compartments by social pressure like parties with friends, peers and executive meetings.

In this paper, we analyze quantitative model of a liquor habit transmission in a population similar to SEIRmodel. The notations are described in Section 2. The mathematical model and basic reproduction number are formulated in Section 3. The local and global stability are derived in Sections 3.2.1 and 3.2.2 respectively. Numerical simulations are illustrated in Section 4 using State-Space model. Discussions and conclusions are given in Section 5.

\section{Notations}

The model is derived using following notations. 
$S(t)$ : the portion of the susceptible who has never liquored;

$E(t)$ : the portion of the total population who liquor less than 2 pegs/day;

$I(t)$ : the portion of the total population who liquor more than 2 pegs/day;

$R(t)$ : the portion of the total population who left liquoring;

$\mu$ : birth rate;

$\beta$ : transmission rate due to the social pressure to adopt liquor habit;

$\alpha$ : rate at which an excessive liquored become normal liquor;

$\rho$ : rate at which ex-liquored returned to liquor;

$c$ : rate at which normal liquored becomes excessive liquor;

$\lambda$ : rate at which normal liquored stop liquoring;

$\gamma:$ rate at which excessive liquored stopping liquoring.

\section{Mathematical Model}

Anybody in the population is susceptible to liquor. In general, one starts taking pegs and increases it gradually. The consumption of two pegs is considered as a normal which falls in E-compartment. The work stress, financial stress, and many more factors to go for more than two pegs which fall under $I$-compartment. Certain fraction of population from $E$ - and $I$-compartments may be removed. It is taken as $R$-compartment. Thus, it resembles toSEIR-model.

Let us call $S, E, I$ and $R$ the portion of total population of each class. Now, we make SEIR-model with some assumptions. The portion of liquored person's who increases liquor habit at a rate proportional to $\beta S(E+I)$ with $\beta>0$. So, the portion of liquored will decrease with the some rate. Here, $\beta$ is called the effective liquoring rate. The portion of liquor person's starting liquoring habit with a rate $\rho R$ (progression rate) with $\rho>0$ and $\alpha I$ with $\alpha>0$. The portion of excessive liquored with the rate $c$ with $c>0$. The portion of removing liquor habit with the rate $\gamma I$ with $\gamma>0$ and $\lambda E$ with $\lambda>0$. See the transfer diagram given in Figure 1.

From Figure 1, the model is formulated as following system of differential equations.

$$
\begin{aligned}
& \frac{\mathrm{d} S}{\mathrm{~d} t}=\mu(1-S)-\beta S(E+I) \\
& \frac{\mathrm{d} E}{\mathrm{~d} t}=\beta S(E+I)+\rho R+\alpha I-(c+\lambda+\mu) E \\
& \frac{\mathrm{d} I}{\mathrm{~d} t}=c E-(\alpha+\gamma+\mu) I \\
& \frac{\mathrm{d} R}{\mathrm{~d} t}=\gamma I+\lambda E-(\rho+\mu) R \\
& \text { with } S+E+I+R=1 \text { and } S>0, E \geq 0, I>0, R \geq 0
\end{aligned}
$$

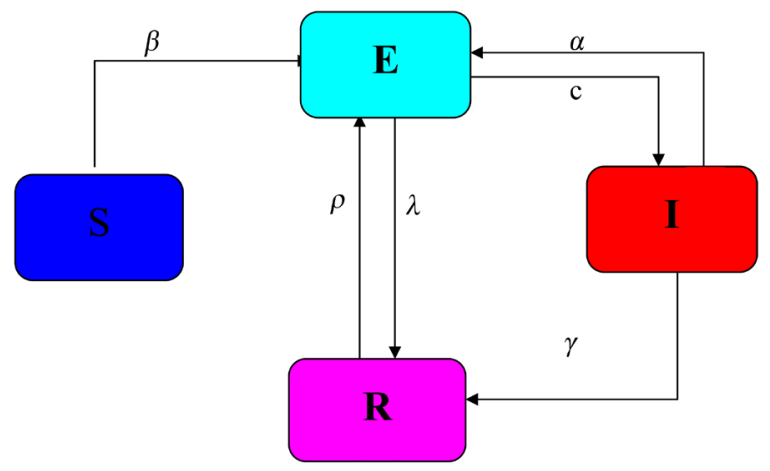

Figure 1. Transfer diagram. 
Since, an epidemic model occurs in a short time period, we ignore moving portion of removal from liquor. So, we will analyze the first three equations forming new reduced system

$$
\begin{aligned}
& \frac{\mathrm{d} S}{\mathrm{~d} t}=\mu(1-S)-\beta S(E+I) \\
& \frac{\mathrm{d} E}{\mathrm{~d} t}=\beta S(E+I)+\rho R+\alpha I-(c+\lambda+\mu) E \\
& \frac{\mathrm{d} I}{\mathrm{~d} t}=c E-(\alpha+\gamma+\mu) I \\
& \text { with } S>0, E \geq 0, I>0
\end{aligned}
$$

Adding all these three equations, we have

$$
\frac{\mathrm{d}}{\mathrm{d} t}(S+E+I)=\mu-\rho R-\lambda E-\gamma I-\mu(S+E+I) \leq \mu-\mu(S+E+I) \geq 0
$$

Which gives, $\lim _{t \rightarrow \infty} \sup (S+E+I) \leq 1$.

Therefore, the feasible region for (2) is

$$
A=\{(S+E+I) / S+E+I \leq 1, S>0, E \geq 0, I>0\} .
$$

Now, the basic reproduction number $R_{0}$ will be found by using the next generation matrix. It is easy to see that (2) always has a liquor free equilibrium $X_{0}=(1,0,0)$.

Let $X^{\prime}=(E, I, S)^{\prime}$, where dash denotes derivative. So that

$$
X^{\prime}=\frac{\mathrm{d} X}{\mathrm{~d} t}=\mathfrak{J}(X)-v(X)
$$

where $\mathfrak{I}(X)$ denotes the rate of appearance of new liquored in compartment and $v(X)$ represents the rate of transfer of liquors, which is given as

$$
\begin{array}{cc}
\mathfrak{I}(X)=\left[\begin{array}{cc}
\beta S(E+I) \\
0 \\
0
\end{array}\right] & v(X)=\left[\begin{array}{c}
-\rho R-\alpha I-(c+\lambda+\mu) E \\
-c E+(\alpha+\gamma+\mu) I \\
-\mu(1-s)+\beta S(E+I)
\end{array}\right] \\
D \Im\left(X_{0}\right)=\left[\begin{array}{ll}
F & 0 \\
0 & 0
\end{array}\right] & D v\left(X_{0}\right)=\left[\begin{array}{ll}
V & 0 \\
J_{1} & J_{2}
\end{array}\right]
\end{array}
$$

$F$ and $V$ are $2 \times 2$ matrices defined as

$$
F=\left[\frac{\partial \Im_{i}\left(X_{0}\right)}{\partial X_{j}}\right] \quad V=\left[\frac{\partial v_{i}\left(X_{0}\right)}{\partial X_{j}}\right] \text { for } i, j=1,2 .
$$

So

$$
F=\left[\begin{array}{cc}
\beta & \beta \\
0 & 0
\end{array}\right] \quad V=\left[\begin{array}{cc}
c+\lambda+\mu & -\alpha \\
-c & \alpha+\mu+\gamma
\end{array}\right]
$$

where $V$ is non-singular matrix, so that

$$
F V^{-1}=\frac{1}{(c+\lambda+\mu)(\alpha+\mu+\gamma-\alpha c)}\left[\begin{array}{cc}
\beta(\alpha+\mu+\gamma+c) & \beta(\alpha+\mu+\gamma+c) \\
0 & 0
\end{array}\right]
$$

Hence, the basic reproduction number $R_{0}$ is

$R_{0}=$ spectral radius of matrix $F V^{-1}$.

$$
R_{0}=\frac{\beta(\alpha+\mu+\gamma+c)}{(c+\lambda+\mu)(\alpha+\mu+\gamma-\alpha c)}
$$


Next, we need to discuss equilibrium of the liquor habit system.

\subsection{Equilibrium}

The liquor free equilibrium is locally asymptotically stable if all the eigenvalues of the matrix have positive real values (Al-Amoudi et al. (2014) [1]).

Theorem 1 (Johnson (2004) [2]): Consider the liquor transmission model given by (2) with $X^{\prime}$. If $X_{0}$ is a liquor free equilibrium of the model, then $X_{0}$ is locally asymptotically stable if $R_{0}>1$, and unstable if $R_{0}<1$, where $R_{0}$ is given by (4).

Proof Let $j=F-V$. Since $V$ is a non-singular matrix and $F$ is non-negative,

Thus $-j=-F+V$ has the $Z$-sign pattern.

$S(j)<0 \Leftrightarrow-j$ isa non-singular matrix. $\{S(j)$ is spectral abscissa of $j\}$

Since $F V^{-1}$ is non-negative, also $-j V^{-1}=1-\left(F V^{-1}\right)$ has the $Z$-sign pattern.

Then, we have $-j$ is a non-singular matrix $\Leftrightarrow 1-\left(F V^{-1}\right)$ is a non-singular matrix.

Finally, since $F V^{-1}$ is non-negative, all eigenvalues of $F V^{-1}$ have magnitude less than or equal to $R_{0}$.

Thus, $1-\left(F V^{-1}\right)$ is a non-singular matrix $\Leftrightarrow R_{0}<1$.

Hence, $S(j)<0$ if and only if $R_{0}<1$.

Similarly, it follows that $S(j)<0 \Leftrightarrow-j$ is a singular matrix.

$\Leftrightarrow 1-\left(F V^{-1}\right)$ is a singular matrix.

$\Leftrightarrow R_{0}=1$

Hence, $S(j)=0$ if and only if $R_{0}=1$.

It follows that $S(j)>0$ if and only if $R_{0}>1$.

\subsection{Stability of the Equilibrium}

\subsubsection{Local Stability}

The liquor free equilibrium is stable if all the eigenvalues of the Jacobian matrix of the system (1) have negative real parts. For this, the Jacobian of the system (1) at $X_{0}=(1,0,0)$ takes the form

$$
J=\left[\begin{array}{cccc}
-\mu & -\beta & -\beta & 0 \\
\beta & \beta-c-\mu-\lambda & \alpha & \rho \\
0 & c & -\alpha-\mu-\gamma & 0 \\
0 & \lambda & \gamma & -\mu-\rho
\end{array}\right]
$$

Here $\operatorname{trace}(J)=-(4 \mu+\alpha-\beta+\gamma+c+\lambda+\rho)<0$.

\subsubsection{Global Stability}

The liquor free equilibrium is globally stable if $1-\left(F V^{-1}\right)>0$.

$$
1-F V^{-1}=I-\frac{1}{|V|}[V]=\frac{|V| I-[V]}{|V|}>0
$$

where $F V^{-1}=\frac{1}{(c+\lambda+\mu)(\alpha+\mu+\gamma-\alpha c)}\left[\begin{array}{cc}\beta(\alpha+\mu+\gamma+c) & \beta(\alpha+\mu+\gamma+c) \\ 0 & 0\end{array}\right]$.

\section{Numerical Simulation}

In this section, we perform numerical simulation of the system (1) using with the state-space model.

\section{State-Space Model}

State equation $\dot{x}=f(x, u)$

Output equation $y=g(x, u)$ where $u$-input, $y$-output, $x$-state vector.

The general state-space description for a linear time invariant, continuous time dynamical system is 


$$
\begin{aligned}
& \dot{x}=A x+B u \\
& y=C x+D u
\end{aligned}
$$

where $A, B, C, D$ are matrices, $\dot{x} \in R^{n}, y \in R^{m}, u \in R^{p}$. Using this state-space model, we find the solution of the system (1). Taking data as follows:

$$
\begin{gathered}
\mu: 0.01 / \text { week } \\
\gamma: 0.0425 / \text { week } \\
\rho: 0.0425 / \text { week } \\
\text { in } A=\left[\begin{array}{cccc}
-\beta & -\beta & -\beta & 0 \\
\beta & -c-\lambda-\mu & \alpha & \rho \\
0 & c & -\alpha-\gamma-\mu & 0 \\
0 & \lambda & \gamma & -\mu-\rho
\end{array}\right] \quad B=\left[\begin{array}{l}
1 \\
0 \\
0 \\
0
\end{array}\right] \quad C=\left[\begin{array}{llll}
1 & 0 & 0 & 0 \\
0 & 1 & 0 & 0 \\
0 & 0 & 1 & 0 \\
0 & 0 & 0 & 1
\end{array}\right] \text { and } D=\left[\begin{array}{llll}
0 & 0 & 0 & 0
\end{array}\right] \text {. }
\end{gathered}
$$

We carry out the simulation. The results are shown in Figure 2.

\section{Discussion and Conclusions}

From Figure 2(a), it is observe that $11 \%$ of the population from the susceptible compartment start liquoring exponentially in first five weeks. Liquoring habit in the susceptible class is small initially but thereafter it increases exponentially. Figure 2(b) is about those who are taking two or less than two pegs in 10 weeks which shows uniform increase, while $44 \%$ of the population is getting liquored in infectious class (Figure 2(c)). It is observed

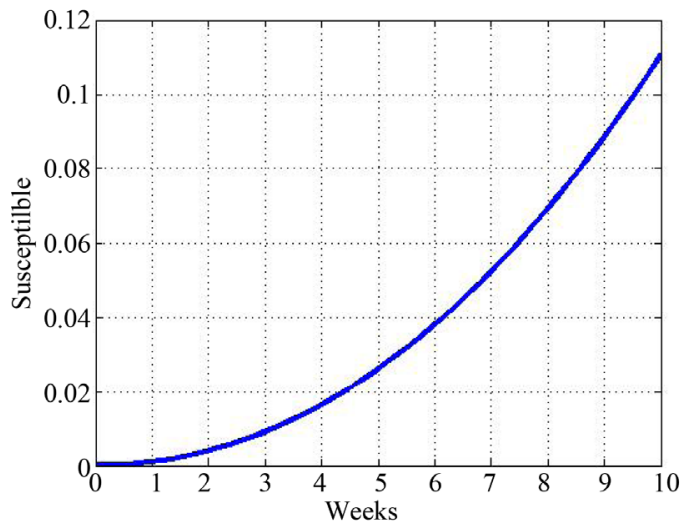

(a)

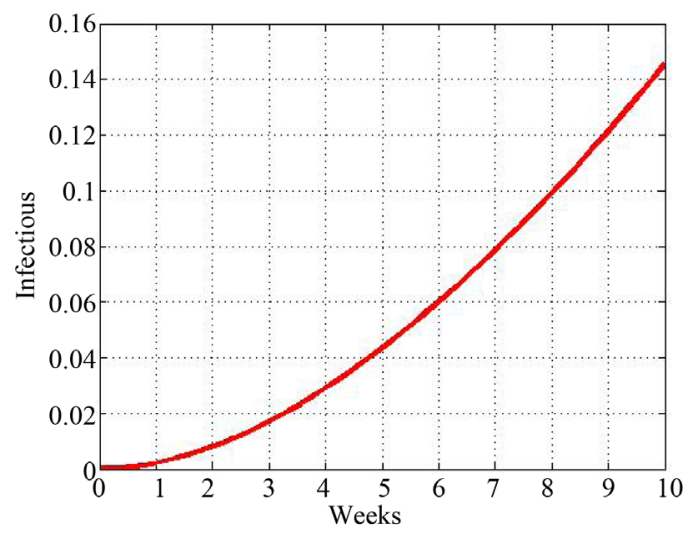

(c)

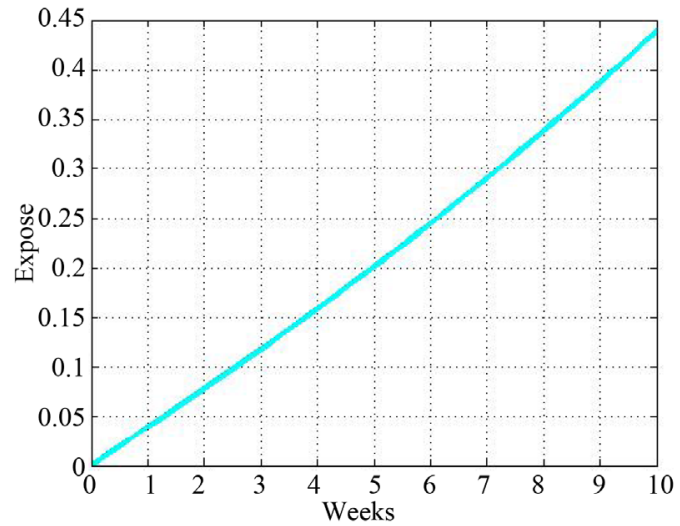

(b)

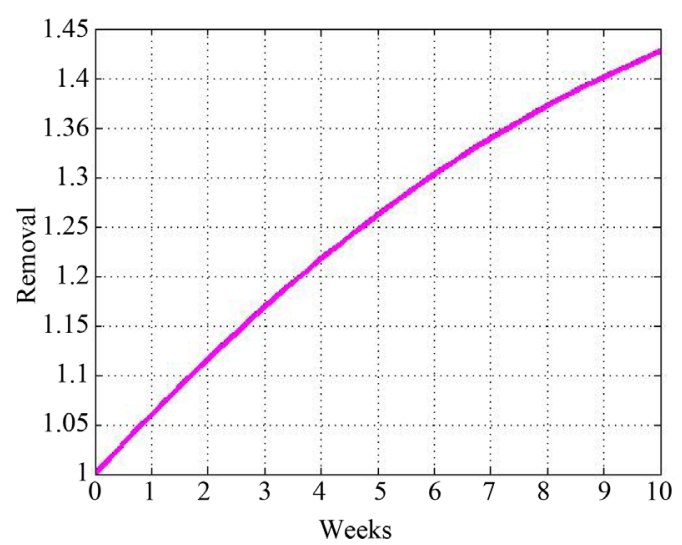

(d)

Figure 2. (a) Susceptible vs weeks; (b) Expose vs weeks; (c) Infectious vs weeks; (d) Removal vs weeks. 
that more than two pegs are taken immediately after $3^{\text {rd }}$ week and increases exponentially. Almost $15 \%$ gets chain liquor (those who can't survive without liquor). The removal compartment (Figure 2(d)), 44\% enters either into less than two pegs i.e. E-compartment or into never liquor that is $S$-compartment.

In this paper, a nonlinear mathematical model for liquor transmission is analysed. The local and global stability of the liquor free equilibrium point are established. It is proved that the free equilibrium is locally asymptotically stable when basic reproduction number $R_{0}<1$ and global stability of liquor transform $1-F V^{-1}>0$.

This model can be extended by educating youth for non-liquoring via advertisement, rehabilitation centre etc.

\section{Acknowledgements}

The first author thanks DST-FIST file \# MSI-097 for technical support.

\section{References}

[1] Al-Amoudi, R., Al-Sheikh, S. and Al-Tuwairqi, S. (2014) Behavior of Solutions to a Mathematical Model of Memes Transmission. International Journal of Applied Mathematical Research, 3, 36-44.

[2] Johnson, L. (2004) An Introduction to the Mathematics of HIV/AIDS Modelling. Centre for Actuarial Research. 